\title{
Effect of Polyuria on Bladder Function in Diabetics versus Non-Diabetics: An Article Review
}

\author{
Ali Fathollahi Firouz Daneshgari Ann T. Hanna-Mitchell \\ Urology Institute, Case Western Reserve University, Cleveland, $\mathrm{OH} .$, USA
}

\section{Key Words}

Polyuria $\cdot$ Diabetes $\cdot$ Bladder

\begin{abstract}
Objectives: To review studies that investigated the role of polyuria on bladder function. Methods: We performed a search of the English literature through PubMed. We only included animal studies that assessed parameters of bladder function and had compared diabetic and non-diabetic polyuric animals. Results: Fluid intake and urine output were increased in diabetic and polyuric animals; failure to appropriately gain weight was seen in diabetics only. All but 1 study reported increase in bladder weight in both groups. In cystometrograms, control mice showed reductions in basal bladder pressure over time, whereas diabetic and diuretic groups stabilized or increased. Both groups showed larger bladder capacity. Overall, many characteristic changes in cystometrographic studies in diabetic animals could be attributed to polyuria. In histological studies, bladder hypertrophy, increase in smooth muscle and urothelium, and increase in protein and DNA content per bladder were observed in diuretic and diabetic rats. Actual collagen crosssectional area did not change during the progression of diabetes or diuresis but decreased over time in both groups as a percentage of total tissue area. Both groups expressed less type I collagen mRNA and TGF-beta-1 mRNA. Conclusions: Although lower urinary tract changes in anatomy and function in diabetic patients might arise from a number of causes, polyuria seems to play an important causative role.
\end{abstract}

Copyright $\odot 2014$ S. Karger AG, Basel

KARGER

Fax +4161306 1234

E-Mail karger@karger.ch

www.karger.com
(C) 2014 S. Karger AG, Basel

1015-9770/14/0083-0119\$38.00/0

Accessible online at:

www.karger.com/cur

\section{Introduction}

Diabetes mellitus (DM) is a metabolic disorder that is characterized by defects in insulin secretion and/or insulin action, resulting in hyperglycemia [1]. Centers for Disease Control and Prevention estimated in 2010 that diabetes affects 25.8 million people in the U.S., $8.3 \%$ of the population, including 7.0 million diabetic individuals who were undiagnosed [2]. Urologic complications, including diabetic bladder dysfunction (DBD), sexual dysfunction, and urinary tract infections, are among the most common, totally accounting for over $50 \%$ of diabetic individuals [3]. Among those, the most common urologic complication of diabetes is DBD $[4,5]$ or diabetic cystopathy [6-11].

Polyuria is one of the most prevalent and first presenting symptoms in diabetic patients. Although there have been many studies on effects of diabetes on bladder function and anatomy, the role of polyuria as an independent factor has been assessed only by a small number of studies.

In the current study, we reviewed the results of studies which were carried out to investigate the role of polyuria on bladder function. We performed a search of the English literature through PubMed. The key words used were "polyuria" or "diuresis", "diabetes" and "bladder dysfunction". We only included those that had been done on animals, assessed parameters of bladder function, and compared diabetic and non-diabetic polyuric animals. We excluded human studies. Fourteen articles were re- 
viewed totally. Our review of the published data and perspective are provided for consideration of the future direction of research.

\section{Materials and Methods}

\section{Animals}

Male Sprague-Dawley rats were used in 11 studies, however, female rats were studied in 1 study; also, 1 study used mice and a rabbit was involved in another. Animals received free access to food and water, except when indicated.

\section{Induction of Diabetes}

In all studies diabetes was induced in the rats with a single injection of streptozotocin $(60-65 \mathrm{mg} / \mathrm{kg}$, intraperitoneally or via the lateral tail vein) in ice-cold 0.01-0.02 M sodium citrate buffer. Successful induction of diabetes was assessed by decreases in rat weight and confirmed by measurement of serum glucose concentration. Rats were assessed 1 day to 20 weeks after the induction of diabetes, according to study design. In 1 study focusing on long-term effects of diabetes, rats were studied for 6 months.

\section{Polyuric Group}

Rats were given $5 \%$ sucrose in tap water to drink instead of water. This was continued until the day of experimentation. Rats were used 1 day to several weeks after beginning sucrose treatment, based on study design. The remaining group of control rats received tap water to drink.

Catheter Implantation and Conscious Cystometrography (CMG)

In 8 studies, either an in vivo and/or in vitro $\mathrm{CMG}$ were done. A suprapubic bladder catheter was implanted. A small incision was made in the bladder wall and the catheter was implanted and fixed. The distal end of the tubing was sealed and the incisions were closed. CMG was performed a few days later according to study design. The bladder was filled via a catheter with $0.9 \%$ saline, while bladder pressure was recorded. Number of micturition cycles differed, but the means were calculated to analyze CMG parameters, including intercontraction interval, peak pressure and voided volume. In addition, functional capacity and bladder compliance were calculated.

\section{Results}

\section{Fluid Intake}

In all studies, there was an increase in fluid intake in diabetic and sucrose-drinking groups [12-17]. Moreover, when this trend was examined more accurately, gradual increases in water consumption and urine excretion in diabetic rats were observed during the first 2 weeks after onset of diabetes [12]. Following streptozotocin, 24-h diuresis increased rapidly to stabilize within 2 weeks at a level 15 times higher than the original. This was ac- complished initially by an increase in the micturition frequency and then gradually by an increased micturition volume [18]. A different pattern was observed with sucrose-drinking rats, where maximal fluid consumption and excretion occurred during the first day after consumption of sucrose solution. The amount of fluid consumed and excreted declined slightly during the next 2 weeks, but then gradually increased back to maximal values at 1 and 2 months [12].

\section{Urine Output}

The average 24-h urine output for sugar-fed and diabetic animals was several folds greater than control. While studies showed similar increasing trends in both groups, the data on urine output comparison between diabetic and sucrose-drinking group was not completely consistent.

A few studies showed that in long term, volumes increased to a much lesser extent in the sucrose-drinking rats than in the diabetics $[12,15,19]$, although still significantly greater than control values after 14 days of treatment [12]. Levin et al. [14] therefore suggested that functional bladder capacity is under neuronal regulation and the change in capacity is not a sole function of the increased bladder mass which occurs at a later time.

The pace of this increase in urine volume occurs has been investigated. Tammela et al. [12] showed that volumes are not significantly increased compared with control until 7 to 14 days after start of sucrose treatment. Daneshgari et al. [19] also showed that the 24-h urine output of the diuretic rats increased after week 3 and became stable after week 9. Interestingly, Tammela et al. [12] found significant differences compared with controls as soon as the first day after induction of diabetes, however, Levin et al.'s results were different in that the increase in micturition volume developed more gradually albeit in a parallel fashion with diuretic group [14].

In more details, Tammela et al. [12] described that after induction of diabetes there were gradual increases in mean and maximal micturition volumes in diabetic rats, with loss of the light-dark cycle differences. Similar increases in mean volume were observed in sucrose-drinking rats, but they were much slower to develop, and the light-dark cycle differential was retained after the first few days.

Levin et al. [14] showed that the magnitude of the increases in micturition frequency and micturition volume paralleled the increase in the total urine volume excreted in the group of sucrose-induced diuresis. 
In conjunction with these observations, Kudlacz et al. [13] showed an enlarged urinary bladder containing a relatively large volume of residual urine on dissection of diabetic rats.

\section{Body Weight}

There was a significantly lower weight gain or even weight loss in diabetic rats as early as 8 weeks after induction of diabetes which persisted after 20 weeks [19]. On initiating insulin treatment, the diabetic rats showed accelerated growth with the rate of weight gain being greater than age-matched controls $[12,13,16,17,19$ 23].

Although weight loss or failure to appropriately gain weight has been observed in diabetic group, consistent lack of difference between diuretic rats and control group in studies seems to exclude the role of polyuria as a contributing factor in weight change.

\section{Bladder Weight}

Except for a study by Malmgren et al. [18] that showed no difference in bladder weight in diabetic rats, all studies reported increases in bladder weight in both the diabetic and polyuric animals [12-17, 19-22, 24, 25]. Tammela et al. [12] showed that the increase became significantly different within 7 days in the diabetic group, but by 30 days polyuric rats could catch up with bladder weight observed in diabetic rats; the bladder weights were not different between polyuric and diabetic rats. Levin et al. [14] also showed the same increasing pattern in bladder mass in diabetic and polyuric rats, but according to their findings, the increase in bladder weight was more gradual in diabetic rats. Malmgren et al. [18] described that after 6 weeks bladder weight increased more than twofold and did not increase further with time. Furthermore, Gray et al. [20] calculated bladder weights normalized to the body weights. They saw a continuously increasing ratio over the experimental time period with significant differences in diabetic (by week 4) and diuretic (by week 8) rats. These findings suggest that although bladder weight might not increase after week 8 , the bladder to body weight ratio continues to increase over a more prolonged period.

Eika et al. [16] and Malmgren et al. [24] studied hereditary polyuric and diabetes insipidus (DI) rats, respectively. Their results showed that bladder mass increased in these animals as well.

Interestingly, when Fukomoto et al. [21] removed sucrose from the drinking water of the sucrose-fed rats after 8 weeks, it resulted in a decrease in bladder weight. Sim-

Polyuria and Bladder Function in Diabetics ilarly, insulin treatment of the diabetic rats diminished the bladder dome weight, although much less compared with polyuric group (from 200 to $123 \%$ versus from 176 to $140 \%$ ).

\section{CMG Results}

Kudlacz et al. [13] showed that in vitro plateau pressure and fluid capacity of bladders in diabetic and diuretic rats were significantly different from control but not from one another. However, according to Daneshgari et al. [19] basal bladder pressure changed similarly for diuretic and diabetic groups, but the diuretic group had greater pressure than the diabetic group. In their study, control mice showed reductions in basal pressure over time, whereas diabetic and diuretic groups stabilized or increased.

During in vitro $\mathrm{CMG}$ derived from diuretic and diabetic animals, transient increases in pressure $(>1$ $\mathrm{cmHg}$ ) were frequently noted in bladder. Treated animals showed a much slower rate of strain measurement, which progressively increased as a function of volume [13].

From the various parameters measured in in vivo CMG, 3 patterns of results were observed. Compared with controls, the polyuric group was characterized by a large volume of fluid required to elicit first contraction, low contraction frequency, a small reduction in contraction duration, and a small increase in intercontraction interval. The diabetic group was similar to controls for the volume to elicit first contraction; it was similar to diuretic group for reduction in contraction frequency, but had a much longer intercontraction interval and shorter contraction duration than other groups [13].

Daneshgari et al. [19] showed that bladder capacity in diabetic and diuretic rats were significantly larger than control and insulin-treated diabetic group at all time points. They confirmed Kudlacz et al.'s [13] findings that diabetic and diuretic groups were not statistically different from one another at all other time points. The diabetic and diuretic groups had also significantly larger compliance levels than the treated diabetic and control groups, but were not different from each other. Wang et al. [22] described that although the compliance of the bladder wall increased progressively and synchronously in both diabetic and diuretic bladders in the first 4 weeks, only the diabetic bladders continued to increase in compliance after 8 weeks. While Eika et al. [16] found similar increase in bladder compliance in hereditary DI, surprisingly, Malmgren et al. [24] observed no significant change in bladder compliance in DI rats, although they noticed lower than normal basal bladder pressure and threshold pressure readings. 
Santicioli et al. [25] studied the changes in threshold pressure and did not find any change in diabetic rats. They deduced that with regard to DBD, increased diuresis has a detrimental and persistent role in increased compliance with other pathophysiologic factors coming into play later on [24].

Level of threshold pressure for the diabetic and diuretic rats were not significantly different from controls in Daneshgari et al.'s study [19], although the pattern of change in threshold pressure differed between control and diabetic groups. The mean intercontraction interval for the control rats increased or remained stable. This pattern differed from that in diabetic and diuretic groups, which had similar trends.

In Daneshgari et al.'s [19] study, mean peak voiding pressure changed over time differently for diabetic rats compared with others. Initially, the peak pressure increased in parallel in diabetic and diuretic rats and was higher than control, but after week 12 it sharply diminished only in diabetic animals. In addition, diabetic rats showed increased resting pressure over time; diuretic and control groups exhibited decreasing trends. As expected, residual urine was highly elevated in diabetic group relative to other groups. This decline in the emptying ability of the bladder was seen in diabetic rats after week 12. Long-term insulin replacement effectively reversed most changes in bladder function. They suggested that the transition from a compensated to a decompensated bladder dysfunction occurs 9-12 weeks after induction of diabetes.

\section{Response to Field Stimulation}

There has been a variety in findings with regard to response of bladder strips to electrical field stimulation. Carpenter [23] reported that nerve-induced bladder contractions developed substantially less pressure in polyuric, diabetic or DI rats than in controls. Contractions elicited at $1 \mathrm{~Hz}$ by control and impaired bladders were potentiated equally by a potassium channel blocker, tetraethyl ammonium chloride (TEA), or by carbachol, an acetylcholine receptor agonist. At $20 \mathrm{~Hz}$, however, contractions by normal bladder strips were not potentiated while those by impaired bladders were. TEA, by increasing transmitter release, and carbachol, by a postjunctional action, substantially reversed bladder dysfunction. Because control and impaired bladders were equally enhanced by TEA, prejunctional and contractile element activity at $1 \mathrm{~Hz}$ were probably unaffected by distension. However, postjunctional sensitivity was probably reduced. Impaired bladders, more compliant than controls, became less compliant after carbachol without elevating resting pressure. He concluded that whereas the action of carbachol to enhance bladder responsiveness did not involve tension development, there may have been cholinoceptor facilitation and shortening of contractile elements.

Eika et al. [16] studied the contractile function using a whole bladder model. Responses of whole bladders from control and diabetic rats to electrical field stimulation, carbachol and $\mathrm{KCl}$ were identical. Volume-pressure relations of the isolated whole bladder showed that the magnitude of the contractile response to $\mathrm{KCl}$ is constant at intravesical volumes ranging from about 10 to $95 \%$ of cytometrical bladder capacity. Bladders from DI rats and diabetic rats showed a rightward shift of volume-pressure curves when compared with controls. Bladders from diuretic rats had volume-pressure curves similar to the bladders from controls. They concluded that while contractile function remains intact with increased diuresis, the passive function changes, with the bladder becoming more distensible.

A more recent study by Tammela et al. [12] showed different results; by 7 days there were significant increases in the maximal contractile responses to carbachol in bladder strips of diabetic rats. The increases in contractility were much slower to develop in diuretic rats and were only significant after 60 days. Response of strips from diabetic rats to $\mathrm{KCl}$ was also significantly greater than those of age-matched controls, while response of strips from diuretic rats was very variable. Because the increased responsiveness occurred so rapidly in diabetic bladders, they believed that early changes were unlikely to result from diabetic neuropathy.

To make it even more confusing, Malmgren et al. [24] found a lower maximal response to $\mathrm{K}(+)$-solution stimulation in bladder strips from DI rats compared with controls. At low frequencies, diabetic bladders exhibited a higher resistance to scopolamine (muscarinic receptor antagonist), but at high frequencies the decrease in resistance observed with aging was similar to controls. Furthermore, the effects of muscarinic blockade were found to be more pronounced in the older control rats [18]. However, Malmgren et al. [24] showed that when activated by field stimulation, the DI and control bladder strips had similar sensitivity to muscarinic receptor blockade with scopolamine at all stimulation frequencies. Malmgren et al. [18], in their previous study, had already shown that the response to field stimulation was reduced in diabetic and increased in diuretic animals. Also, in contrast to diabetic rats, the sensitivity to carbachol 
was similar in DI and control groups [24]. Their results opposed Eika et al.'s [16] in which diabetic rats showed similar responses to carbachol as the control group. They suggested that the increased functional demands of DI on the detrusor did not result in major changes pre- or post-junctionally [24]. This was in support of their previous suggestion that increased resistance to muscarinic receptor blockade in diabetic rats at low stimulation frequencies was induced by the disease (DM) and not by increased diuresis [18].

Reserpine administration, effects of which include depletion of norepinephrine in sympathetic nerves, reduces the volume to first contraction, decreases contraction frequency, reduces the duration of contractions, and lengthens the intervals between contractions. Because the pattern of in vivo CMG from diabetic animals resembles that from reserpine-treated animals more closely than those from other groups, additional experiments were performed by Kudlacz et al. [13] with this condition. Reserpine pretreatment had smaller effects in diabetic animals, whereas it produced quantitatively similar changes in diuretic and control animals for all parameters except volume to first contraction. They suggested that diabetic bladders were lacking sympathetic control before drug treatment. When diuretic rats were removed from their diet for 4 weeks, the bladders responded similar to controls. This observation, coupled with the fact that their bladder did not produce the same response as diabetics in vivo, suggests that the diuretic model does not produce irreversible functional neuropathies.

Contents of urinary bladder and ureters in sensory neuropeptides (substance P, neurokinin-A and calcitonin-gene related peptide-like immunoreactivity) were assessed by Santicioli et al. [25]. They showed an increase in those values in diabetes even when values were corrected for increased organ weight.

Capsaicin administration produces a specific sensory neuropathy by prolonged activation of neurons which, in turn, depletes presynaptic substance $P$, a pain neurotransmitter. When compared with control, capsaicin treatment results in a very large volume to first contraction, decreased contraction frequency, no change in the duration of contractions, and lengthened intervals between contractions. Santicioli et al. [25] showed that capsaicin-induced contraction of rat isolated bladder strips, presumably caused by neuropeptides released from intramural sensory nerves, was unaffected by diabetes. They indicated that diabetes produces, at an early stage, changes similar to those reported to occur in the human disease, e.g. a greater bladder capacity with unimpaired voiding

Polyuria and Bladder Function in Diabetics function. They suggested that increased bladder capacity of diabetic rats could mainly be attributed to changes in physical properties of the detrusor muscle, thereby allowing accommodation of greater than normal volumes with similar increase of intraluminal pressure.

\section{Bladder Histology}

In the study by Kudlacz et al. [13], the increase in organ weight was associated with an increase in protein and DNA content per bladder for polyuric and diabetic rats, but when protein and DNA were normalized to bladder weight, no difference was found.

Liu et al. [15] observed decreased bladder wall thickness in diabetic and diuretic rats within 2 weeks which then gradually returned to control values at 3 and 9 weeks. The lumen areas were greater in diabetic and diuretic rats at 9 weeks. The total cross-sectional area of the bladder wall of diabetic and diuretic rats also increased rapidly after induction and continued to increase thereafter. This was confirmed by another study by Xiao et al. [17]. Total cross-sectional area of bladder lumen (at the equatorial midline) increased significantly as soon as 4 days after induction in diabetic and diuretic rats and then gradually increased until 3 weeks, with no further increase at 9 weeks [15]. There was no significant difference in lumen area, wall area, or wall thickness between diabetic and diuretic rats at any time [15]. As described earlier in bladder weight, bladder hypertrophy was obviously seen in diabetic and diuretic groups [17, 24].

Liu et al. [15] also analyzed relative content of the tissue components of bladder wall. The absolute crosssectional area of urothelium increased gradually in diabetic and diuretic rats. However, when expressed as percentage of the total tissue area, urothelium area was not significantly greater during progression of diabetes and diuresis. Smooth muscle area of the bladder wall and smooth muscle area as a percentage of total tissue area increased significantly in diabetic and diuretic animals. There was no significant difference between diabetic and diuretic rats in areas of any of tissue components at any time [15]. Again, Xiao et al.'s [17] study confirmed an increase in smooth muscle and urothelium in diabetic and diuretic rats. They suggested that the significantly increased bladder wall areas in these rats are due primarily to increased smooth muscle and urothelium [15].

In bladder, collagen is mainly localized in the lamina propria and within and between the muscle bundles. Liu et al.'s [15] results revealed that actual collagen crosssectional area did not change significantly during the progression of diabetes or diuresis but decreased over 
time in diabetic and diuretic groups as a percentage of the total tissue area. Gray et al. [20] measured changes in the expression of collagen subtypes, reporting that diabetic and diuretic rat bladders exhibited significantly lower expression of type I collagen mRNA at all time points tested. In contrast, type III collagen mRNA expression in these animals was time-dependent; for both groups, compared with normal rats, type III collagen mRNA expression was similar in short term but significantly lower after 4 weeks. Eika et al. [16] demonstrated that although diabetic and diuretic rats had decreased collagen content, the collagen concentration of diabetic bladders was significantly lower than that of diuretic and congenital DI rat models, suggesting that the decrease in bladder collagen concentration associated with diabetes was only partly related to increased diuresis. Furthermore, the tropoelastin mRNA expression in diabetic rat bladders was, compared to controls, significantly higher early after diabetes induction but similar later; diuretic rat bladders, conversely, exhibited expression of tropoelastin mRNA similar to that of controls at all time points. In addition, both diabetic and diuretic rat bladders exhibited significantly lower expression of TGF-beta-1 mRNA at all time points. Focusing on the elastin content (normalized by wet tissue weight), diabetic and diuretic bladders contained significantly higher amounts. The results of their study suggest that the increase in bladder compliance in diabetic cystopathy results not only from diuresis-driven reduction of collagen synthesis but also from increased elastin synthesis [20].

Fukomoto et al. [21] identified the presence of a single class of specific, saturable, high-affinity, noninteracting binding sites (muscarinic receptors) in rat bladder domes using saturation experiments with ${ }^{3} \mathrm{H}$-quinuclidinyl benzilate, as a binding assay for rat bladder dome muscarinic receptor. In bladder domes of diabetic and polyuric groups, the density of muscarinic receptors increased significantly. Moreover, the increase was much higher in the diabetic rat bladder than in diuretic animals. Although treatment with insulin in diabetic rats resulted in a significant decrease in the maximum number of binding sites (Bmax) values of bladder dome muscarinic receptors, the Bmax values still remained higher than control rats when expressed as density of receptors. They concluded that, although insulin treatment can, to some extent, reverse diabetes-induced upregulation of muscarinic cholinergic receptors in rat bladder dome, it does not completely correct the receptor abnormality to a normal level. On the other hand, the Bmax for muscarinic cho- linergic receptors in the bladder dome of diuretic rats, which were withdrawn from sucrose drinking water, was similar to control rats.

When binding properties of a muscarinic cholinergic agonist to muscarinic receptors in rat bladder dome was investigated with inhibition experiments in which ${ }^{3} \mathrm{H}-\mathrm{QNB}$ binding was inhibited by increasing concentrations of carbachol, the binding curves for carbachol in all groups were shallow, indicating the presence of more than one affinity constant.

To examine the effect of muscarinic receptor upregulation on possible changes in muscarinic subtypes in rat bladder dome, Fukomoto et al. [21] then performed parallel inhibition experiments with ${ }^{3} \mathrm{H}-\mathrm{QNB}$ with selective muscarinic cholinergic antagonists: pirenzepine, methoctramine, and 4-DAMP. Upregulations of muscarinic receptors associated with diabetes and polyuria did not involve a significant alteration in muscarinic receptor subtype specificity.

The extent of damage due to oxygen radicals is largely determined by the activity of free radical scavengers such as superoxide dismutase (SOD). Manganese SOD (MnSOD), a member of SOD family, is the most important isozyme in DM because mitochondrial respiration is the major source of hyperglycemia induced intracellular reactive oxygen species production. Xiao et al. [17] measured MnSOD and showed its increase in the diabetic group compared with control and polyuric groups.

\section{Conclusion}

Diabetic patients often demonstrate a variety of symptoms affecting the urinary bladder, including bladder hyperreflexia and areflexia and indeterminate or normal bladder function [26, 27]. Although it is yet to be fully understood, this spectrum in manifestations might arise from a number of causes, among which polyuria seems to play a part. 


\section{References}

1 Mokdad AH, Ford ES, Bowman BA, Nelson DE, Engelgau MM, Vinicor F, Marks JS: The continuing increase of diabetes in the US. Diabetes Care 2001;24:412.

2 Centers for Disease Control and Prevention. National diabetes fact sheet: National estimates and general information on diabetes and prediabetes in the United States, 2011. Atlanta, GA, U.S. Department of Health and Human Services, 2011.

3 Brown JS, Wessells H, Chancellor MB, Howards SS, Stamm WE, Stapleton AE, Steers WD, Van Den Eeden SK, McVary KT: Urologic complications of diabetes. Diabetes Care 2005;28:177-185.

4 Baneerjee MK, Das AK, Basha A, Ganesan TS, Chandrasekhar S: Reversibility of diabetic bladder dysfunction with early and good control. J Assoc Physicians India 1985; 33:337-338.

5 Daneshgari F, Liu G, Birder L, Hanna-Mitchell AT, Chacko S: Diabetic bladder dysfunction: current translational knowledge. J Urol 2009;182(6 suppl):S18-S26.

6 Moller CF: Diabetic cystopathy. I: A clinical study of the frequency of bladder dysfunction in diabetics. Dan Med Bull 1976;23:267-278.

7 Moller CF: Diabetic cystopathy. II: Relationship to some late-diabetic manifestations. Dan Med Bull 1976;23:279-287.

8 Moller CF: Diabetic cystopathy. III: Urinary bladder dysfunction in relation to bacteriuria. Dan Med Bull 1976;23:287-291.

9 Frimodt-Moller C: Diabetic cystopathy. A review of the urodynamic and clinical features of neurogenic bladder dysfunction in diabetes mellitus. Dan Med Bull 1978;25:49-60.

10 Kaplan SA, Blaivas JG: Diabetic cystopathy. J Diabet Complications 1988;2:133-139.
11 Liu G, Daneshgari F: Diabetic bladder dysfunction. Chin Med J (Engl) 2014;127:13571364.

12 Tammela TLJ, Leggett RE, Levin RM, Longhurst PA: Temporal changes in micturition and bladder contractility after sucrose dieresis and streptozotocin-induced diabetes mellitus in rats. J Urol 1995;153:2014-2021.

13 Kudlacz EM, Chun AL, Skau KA, Gerald MC, Wallace LJ: Diabetes and diuretic-Induced alterations in function of rat urinary bladder. Diabetes 1988;37:949-955.

14 Levin RM, Wein AJ, Eika B, Tammela TL, Longhurst PA: Effects of diuresis on micturition. Neurourol Urodyn 1995;14:169-176.

15 Liu G, Daneshgari F: Temporal diabetes- and diuresis-induced remodeling of the urinary bladder in the rat. Am J Physiol Regul Integr Comp Physiol 2006;291:R837-R843.

16 Eika B, Levin RM, Longhurst PA: Comparison of urinary bladder function in rats with hereditary diabetes insipidus, streptozotocin-induced diabetes mellitus, and nondiabetic osmotic diuresis. J Urol 1994;151:496502.

17 Xiao N, Wang Z, Huang Y, Daneshgari F, Liu $\mathrm{G}$ : Roles of polyuria and hyperglycemia in bladder dysfunction in diabetes. J Urol 2013; 189:1130-1136.

18 Malmgren A, Andersson PO, Uvelius B: Bladder function in rats with short- and longterm diabetes; effects of age and muscarinic blockade. J Urol 1989;142:1608-1614.

19 Daneshgari F, Huang X, Liu G, Bena J, Saffore L, Powell CT: Temporal differences in bladder dysfunction caused by diabetes, diuresis, and treated diabetes in mice. Am $\mathbf{J}$ Physiol Regul Integr Comp Physiol 2006; 290:R1728-R1735.
20 Gray MA, Wang CC, Sacks MS, Yoshimura $\mathrm{N}$, Chancellor MB, Nagatomi J: Time-dependent alterations of select genes in streptozotocin-induced diabetic rat bladder. Urology 2008;71:1214-1219.

21 Fukomoto Y, Yoshida M, Weiss RM, Latifpour J: Reversibility of diabetes- and diuresis-induced alterations in rat bladder dome muscarinic receptors. Diabetes 1994;43:819826.

22 Wang CC, Nagatomi J, Toosi KK, Yoshimura N, Hsieh JH, Chancellor MB, Sacks MS: Diabetes-induced alternations in the biomechanical properties of the urinary bladder wall in rats. Urology 2009;73:911-915.

23 Carpenter FG: Impairment and restoration of rat urinary bladder responsiveness following distension. Am J Physiol 1983;244:R106113.

24 Malmgren A, Uvelius B, Andersson KE, Andersson PO: Urinary bladder function in rats with hereditary diabetes insipidus; a cystometrical and in vitro evaluation. J Urol 1992;148:930-934.

25 Santicioli P, Gamse R, Maggi CA, Meli A: Cystometric changes in the early phase of streptozotocin-induced diabetes in rats: evidence for sensory changes not correlated to diabetic neuropathy. Naunyn Schmiedebergs Arch Pharmacol 1987;335:580-587.

26 Kaplan SA, Te AE, Blaivas JG: Urodynamic findings in patients with diabetic cystopathy. J Urol 1995; 153:342-344.

27 Starer P, Libow L: Cystometric evaluation of bladder dysfunction in elderly diabetic patients. Arch Intern Med 1990;150:810-813. 